\title{
O CONTROLE GLICÊMICO ESTRITO PIORA O RISCO CARDIOVASCULAR NO DIABETES TIPO 2?
}

Complicações cardiovasculares são as principais causas de morbidade e mortalidade em pacientes com diabetes tipo 2 ! Mais de $60 \%$ da morbidade, mortalidade e gastos em saúde com pacientes com diabetes tipo 2 são decorrentes de doença cardiovascular, no entanto o papel do controle glicêmico na redução desse risco ainda é incerto. Poucos são os estudos de intervenção que objetivam avaliar se o controle da glicemia melhora o desfecho cardiovascular. Tanto o estudo Diabetes Control and Complications Trial(DCCT) em diabetes tipo $\mathrm{I}^{2} \mathrm{e}$ - United Kingdom Prospective Diabetes Study (UKPDS) em diabetes tipo 2 recém-diagnosticado ${ }^{3}$ mostraram um efeito benéfico significativo nas complicações microvasculares, assim como o estudo de Kumamoto em diabetes tipo 2 insulinizados ${ }^{4}$. O Veterans Affairs Cooperative Study on Glycemic Control and Complications in NIDDM5, estudo em diabéticos tipo 2 insulinizados, no entanto, encontrou uma forte tendência para a piora do desfecho cardiovascular com o controle intensivo.

No dia 6 de fevereiro passado, a comunidade científica envolvida no estudo do diabetes foi surpreendida com a publicação da decisão do National Heart, Lung and Blood Institute de interromper o braço intensivo do estudo ACCORD (Action to control cardiovascular risk in Diabetes) por aumento da mortalidade cardiovascular6. O estudo ACCORD, conduzido pelo National Institute of Health dos Estados Unidos e isento de interesses comerciais, era aguardado com ansiedade. Estavam sendo acompanhados 10.152 pacientes com diabetes tipo 2 e doença cardiovascular ou a presença de dois fatores de risco cardiovasculares. Todos os pacientes testavam se a meta de controle glicêmico (Alc menor que 6\%) era superior que a meta padrão (Alc entre $7 \%$ e 7,9\%) na redução das complicações cardiovasculares. Dependendo da presença de hipertensão arterial e dislipidemia testavam também a meta de PA sistólica inferior a I $20 \mathrm{mmHg}$ vs inferior a $140 \mathrm{mmHg}$ e o controle da dislipidemia com estatina e fibrato ou estatina e placebo. Não havia determinação de droga específica a ser usada, ou seja, poderiam ser prescritas quaisquer medicações que fossem necessárias para controlar a glicemia. O estudo encontrou que no braço intensivo a A I c média foi de $6,4 \%$ e no convencional, de 7,5\%. A mortalidade foi de $14 /$ I000 pacientes/ano no grupo intensivo e de II// 000 pacientes/ ano no grupo convencional, razão para que fosse interrompido o braço intensivo. Na mesma semana, foram publicados os resultados da extensão do estudo Steno- $2^{7}$, que consiste na intervenção multifatorial para analisar desfecho cardiovascular relacionado ao controle da glicemia, da hipertensão, da dislipidemia, da obesidade e do tabagismo. Nesse estudo, de 13,3 anos de acompanhamento o grupo intensivo teve uma redução da mortalidade cardiovascular de $43 \%$ e de eventos cardiovasculares em $41 \%$.

No dia I 4 de fevereiro, o Data Safety and Monitoring Board do estudo ADVANCE ${ }^{8}$ (ainda em andamento) analisou seus dados e não confirmou os mesmos achados do ACCORD. O estudo ADVANCE, conduzido na Austrália, tem um desenho semelhante ao ACCORD, no entanto, tanto o braço de controle intensivo da glicemia quanto o de controle padrão ofereciam a melhor opção para o controle da hipertensão arterial e da dislipemia.
Essa eclosão recente da divulgação de dados aparentemente incoerentes deve servir para algumas reflexões. Devemos distinguir o efeito direto da hiperglicemia na gênese da microangiopatia e o efeito da hiperglicemia na macroangiopatia. A ateroesclerose, base da doença macrovascular isquêmica, tem uma fisiopatologia complexa, com vários fatores etiológicos. Uma conclusão inicial baseada nessas premissas é que os esforços para reduzir a glicemia devem ser balanceados entre os riscos e os benefícios. A consideração para uma terapia intensiva da glicemia deve incluir não somente os benefícios para a microcirculação (retinopatia, nefropatia e neuropatia), mas também para as possibilidades de eventuais complicações como hipoglicemia com implicações físicas, mentais, sociais e econômicas. A segunda reflexão deve lembrar que atingir meta de controle glicêmico estrito pode desencadear ganho de peso com piora da hipertensão arterial e da dislipidemia. $O$ próprio UKPDS ${ }^{9}$ demonstrou uma série de potenciais fatores de risco modificáveis para doença arterial coronariana, sendo em ordem de importância através de análise multivariada as concentrações de LDL colesterol, a diminuição do HDL-colesterol, aumento da pressão arterial, a Al c e o tabagismo. O estudo Steno-2 demonstra que é essencial controlar todos os fatores de risco cardiovasculares. Devemos lembrar ainda que a utilização de vários medicamentos, como no caso do ACCORD, necessita de estudos de segurança da interação medicamentosa. Os pacientes com diabetes tipo 2 devem discutir com seus médicos os riscos e benefícios de qualquer intervenção medicamentosa. Isso seguramente não significa que não devamos atingir metas glicêmicas normais e sim que devemos tratar com visão multifatorial uma doença multifatorial.

Marcos TAMBascia

\section{Referências}

I.Geiss S, Herman WH, Smith PJ. Mortality in non-insulin dependent diabetes In: Harris M, editor. Diabetes in America / National Diabetes Data Group. $2^{a}$ ed Bethesda: National Institute of Health; 1995. p. I33-55.

2. The Diabetes Control and Complications Trial Research Group. The effect of intensive treatment of diabetes on the development and progression of longterm complications in insulin-dependent diabetes mellitus. N Eng J Med. 1993 329:977-86.

3.UK Prospective Diabetes Study (UKPDS) Group. Intensive blood-glucose control with sulfonylurea or insulin compared with conventional treatment and risks of complications in patients with type 2 diabetes (UKPDS 33). Lancet. 1998:352:837-53

4.Shichiri M, Ohkubo Y, Kishikawa H, Wake N. Long-term results of the Kumamoto study on optimal diabetes control in type 2 diabetes mellitus. Diabetes Care. 2000;23 (Suppl 2) B2 I-B29, 2000.

5.Abraira C, Colwell J, Nuttall F, Sawin C, Sawin CT, Henderson W, Comstock $\mathrm{IP}$, et al. The Veterans Affairs Cooperative Study on Glycemic Control and Complications in type 2. Diabetes Group: Cardiovascular events and correlates in the VA Feasibility Trial. Arch Intern Med. 1997; I 57: I 8 | -8.

6. National Heart, Lung and Blood Institute. ACCORD telebriefing prepared remarks [cited 2008 Feb 6]. Available from: http://www.nhlbi.nih.gov/health/ prof/heart/other/accord/index.htm

7. Gaede P, Lund-Anderson H, Parvin $\mathrm{HH}$, Pedersen $\mathrm{O}$. Effect of a multifactorial intervention on mortality in type 2 diabetes. N Engl J Med. 2008;358: 580-I. 8.Advance. Major International Diabetes Study not confirms increased risk of death reported by US Trial [cited 2008 Feb 6]. Available from: http:// www.sciencedaily.com/releases/2008/02/0802 | 4092 I 56.htm.

9. Turner RC. Millns H, Neil HAW, Stratton IM, Manley SE, Matthews DR, et al Risk factors for coronary artery disease in non-insulin dependent diabetes mellitus United Kingdom prospective diabetes study (UKPDS: 23). BMJ. 1998;3 I 6:823-8 